\title{
Quasicrystalline Order in Binary Dipolar Systems
}

\author{
Falk Scheffler, ${ }^{1}$ Philipp Maass, ${ }^{2}$ Johannes Roth, ${ }^{3}$ and Holger Stark ${ }^{1}$ \\ ${ }^{1}$ Fachbereich Physik, Universität Konstanz, 78457 Konstanz, Germany \\ ${ }^{2}$ Institut für Physik, Technische Universität Ilmenau, 98684 Ilmenau, Germany* \\ ${ }^{3}$ Institut für Theoretische und Angewandte Physik, Universität Stuttgart, 70550 Stuttgart, Germany
}

(Dated: April 15, 2004)

\begin{abstract}
Motivated by recent experimental findings, we investigate the possible occurrence and characteristics of quasicrystalline order in two-dimensional mixtures of point dipoles with two sorts of dipole moments. Despite the fact that the dipolar interaction potential does not exhibit an intrinsic length scale and cannot be tuned a priori to support the formation of quasicrystalline order, we find that configurations with long-range quasicrystallinity yield minima in the potential energy surface of the many particle system. These configurations emanate from an ideal or perturbed ideal decoration of a binary tiling by steepest descent relaxation. Ground state energy calculations of alternative ordered states and parallel tempering Monte-Carlo simulations reveal that the quasicrystalline configurations do not correspond to a thermodynamically stable state. On the other hand, steepest descent relaxations and conventional Monte-Carlo simulations suggest that they are rather robust against fluctuations. Local quasicrystalline order in the disordered equilibrium states can be strong.
\end{abstract}

\section{INTRODUCTION}

Since the surprising discovery of sharp diffraction images with non-crystallographic symmetry in some rapidly quenched metal alloys by Shechtman et al. in 1984 [1], quasicrystalline structures have attracted fastly growing interest as an alternative type of structure of solid matter. As a distinctive feature, these quasicrystals possess long-range positional order in combination with a crystallographically 'forbidden' (e.g. fivefold) point group symmetry, which necessarily means aperiodic order. By now, many systems with a quasicrystalline order have been identified in nature, most of them being ternary or, in a few cases, binary alloys (for a review see [2]). Very recently, quasicrystalline structures with fundamental building blocks much larger than single atoms have been found in micellar phases of dendrimers (tree-like molecules) [3]. They represent a new mode of organization in soft matter and are interesting in connection with photonic bandgap materials [4] and photonic quasicrystal lasers [5].

On the theoretical side, the formation of quasicrystals could be reproduced in simulations of binary mixtures with hard sphere or Lennard-Jones potentials [6, 7]. In these simulations the quasicrystalline structures were stabilized by tuning the distances corresponding to the minima of the interaction potentials to match the specific particle-particle distances.

Recently, some evidence for local patters with fivefold symmetry was found in two-dimensional binary mixtures of superparamagnetic colloidal particles [8, 9, 10, 11]. Hence the intriguing question arises: Can there be quasicrystalline long-range order in a binary dipolar system despite the fact that the dipolar interaction potential does not possess tunable intrinsic length scales?

*Electronic address: Philipp.Maass@tu-ilmenau.de
Here, we tackle this problem by investigating the occurrence of quasicrystalline order in binary mixtures of point dipoles with varying dipole strengths. As a twodimensional reference structure with fivefold symmetry, we use the prominent rhombic binary tiling and decorate it with two types of dipoles. Then we let it relax mechanically and find that for a certain range of dipolar strength ratios $D$ the mechanically stable configuration preserves the long-range quasicrystalline order (see sec. IIC below). The final configuration corresponds to a local minimum in the potential energy surface of the many-particle system. However, its global features are still undetermined. It could correspond to the ground state, a thermodynamically stable state within a certain parameter regime (of temperature, mixing ratio and dipolar strength ratio), or a metastable state that, below some freezing temperature, becomes separated from other states by free energy barriers which are infinite in the thermodynamic limit of infinite system size. This is reminiscent to the behavior of mean-field spin-glass models [12]. In both cases, one would expect the dipolar system to evolve in time at some finite temperature and ultimately build up permanent long-range quasicrystalline order if its initial configuration belongs to the attraction basin of the quasicrystalline state. On the other hand, the mechanically relaxed quasicrystal structure could correspond to a metastable state from which the system escapes when it surmounts a finite free energy barrier. Nevertheless, within the last scenario, it would be interesting to see, whether quasicrystalline ordering exists locally in the disordered equilibrium state of the system.

To evaluate the global features as discussed in the last paragraph, we perform a number of investigations. We first compare in sec. III the energy of several plausible alternative ground state structures with the energy of the quasicrystalline state. Then, we assess in sec. IV the stability of the quasicrystalline structure, for varying dipole strength ratio, with respect to small random perturba- 


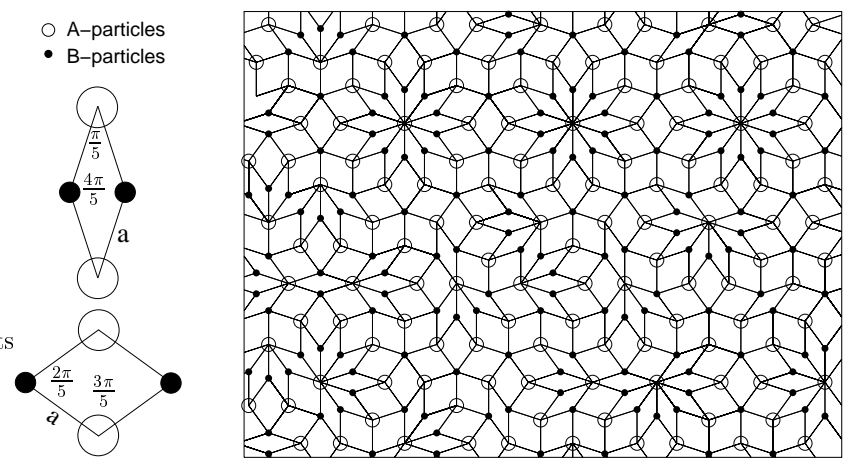

FIG. 1: The binary tiling decorated by strong (A) and weak (B) dipoles. The angles enclosed by the edges of its two rhombic building blocks are all multiples of $\pi / 5$, which gives rise to the non-translation invariant 10 -fold symmetry.

tions of the particle positions in the ideal decoration. Finally, in sec. $\nabla$ we perform Monte Carlo simulations to analyze the characteristics of quasicrystalline order at finite temperatures. Since for the thermalization of the system conventional Monte Carlo techniques turned out to be ineffective, we applied a parallel tempering protocol to reach thermodynamic equilibrium.

\section{MODEL}

\section{A. System parameters and order parameter}

Corresponding to the experimental situation [9, 10], we consider particles moving in a plane with their dipolar moments all pointing in the same direction perpendicular to the plane. In experiments this can be realized by letting superparamagnetic particles float on a liquid meniscus in an external magnetic field.

To investigate quasicrystalline ordering, we choose as a reference structure the two-dimensional binary tiling, which consists of two types of rhombs put together by certain matching rules [13]. It is a typical example for a quasicrystalline pattern with fivefold symmetry (see fig. 1). A crystal results from a periodically repeated unit cell decorated by atoms. Here we obtain the ideal quasicrystalline reference structure by decorating the rhombs as illustrated in fig. 1 and described in ref. 14 so that it consists of $N_{\mathrm{A}}$ strong $\mathrm{A}$ and $N_{\mathrm{B}}$ weak B dipoles. The mixing ratio $x=N_{\mathrm{A}} /\left(N_{\mathrm{A}}+N_{\mathrm{B}}\right)$ is $\tau /(2+\tau) \cong 0.447$, where $\tau=(1+\sqrt{5}) / 2$ is the golden mean. A picture of the final structure is shown in fig. 1. The decoration was already used in previous simulations of binary LennardJones and hard sphere systems [6, 7, 14]. However, in contrast to these simulations, the potential of the dipolar system cannot be optimized in an obvious way to support the formation of the quasicrystalline structure.

The interaction energy of two parallel magnetic mo- ments $m_{1}$ and $m_{2}$ at a distance $r$ is given by

$$
E_{\mathrm{int}}=\frac{\mu_{0}}{4 \pi} \frac{m_{1} m_{2}}{r^{3}}
$$

where $\mu_{0}$ is the vacuum permeability. In the following we switch to dimensionless quantities. As unit length we choose the edge $a$ of a rhomb, i.e., the distance of two neighboring $\mathrm{A}$ - and $\mathrm{B}$-dipoles in the reference structure. As unit of energy (and temperature $T$ ) we choose the interaction energy $\left(\mu_{0} / 4 \pi\right) \cdot\left(m_{A} m_{B} / a^{3}\right)$ of two such dipoles. After introducing the ratio $D \equiv m_{\mathrm{A}} / m_{\mathrm{B}}$ of dipole strengths $m_{\mathrm{A}}$ and $m_{\mathrm{B}}$, the interaction potentials of two A-dipoles, an A-and a B-dipole, and two B-dipoles at distance $r$ are

$$
E_{\mathrm{AA}}=D r^{-3}, \quad E_{\mathrm{AB}}=r^{-3}, \quad E_{\mathrm{BB}}=D^{-1} r^{-3} .
$$

The dimensionless form clarifies that the dipole strength ratio $D$ is the only additional parameter in our problem besides the temperature $T$ and the (fixed) mixing ratio $x$. This is a direct consequence of the scale-free dipolar interaction potential.

In order to quantify the degree of quasicrystalline order, we define the order parameter

$$
\phi=\left|\frac{1}{2 N_{p}} \sum_{j, k} \theta\left(r_{\max }-r_{j, k}\right) \exp \left(i \cdot 10 \alpha_{j, k}\right)\right|,
$$

where $\theta($.$) is the conventional step function (\theta(x)=1$ for $x>0$ and zero else), $N_{p}=\sum_{j, k} \theta\left(r_{\max }-r_{j, k}\right)$ is the number of pairs of dipoles with distances $r_{j, k}$ smaller than $r_{\max } \equiv 1.15$, and $\alpha_{j, k} \equiv \Varangle\left(\mathbf{r}_{j, k}, \hat{\mathbf{e}}\right)$ is the bond angle between the pair vector $\mathbf{r}_{j, k}$ and an arbitrary but fixed direction ê. Since the bond-angles in the binary tiling are all multiples of $\pi / 5$ (cf. fig. प) $\phi=1$ in the ideal quasicrystalline structure, while $\phi=0$ for a system without tenfold bond orientational order. The value $r_{\max }$ is slightly smaller than the distance of the two A particles in the fat rhomb shown in fig. 1 Eliminating these pairs from the sum in eq. (3), on the one hand, allows us to encompass slightly displaced "nearest neighbors" but, on the other hand, guarantees that bond angles different from multiples of $\pi / 5$ are not counted in the ideal quasicrystalline configuration.

In addition to (31), we also consider the $n$-fold local bond orientational order parameters

$$
\tilde{\phi}_{n}=\frac{1}{2 N} \sum_{j} \frac{1}{N_{j}}\left|\sum_{k} \theta\left(r_{\max }-r_{j, k}\right) \exp \left(i \cdot n \alpha_{j, k}\right)\right|,
$$

where $N_{j}=\sum_{k} \theta\left(r_{\max }-r_{j, k}\right)$ is the number of neighbors of dipole $j$. Since the absolute value is taken before averaging over all $N$ dipoles, the $\tilde{\phi}_{n}$ are sensitive to the $n-$ fold symmetric arrangement of nearest neighbors around any dipoles, but insensitive to the spatial variation of the bond orientations. Naturally, we have $\tilde{\phi}_{10} \geq \phi$. 


\section{B. Rational approximants}

Due to the missing translational invariance, standard periodic boundary conditions cannot be imposed to ideal quasicrystalline systems. To resolve this problem, it is convenient to use rational approximants of quasicrystals as described in [15]. A rational approximant is a rectangular part of the ideal quasicrystalline structure which is chosen such that it may be exposed to periodic boundary conditions without too much distorting the local quasicrystalline ordering.

From a number of different rational approximants, we mainly used a small one with 890 dipoles (398 A, 492 B dipoles) and size $24.80 \times 29.15$, and a large one with 1700 dipoles (760 A, 940 B dipoles) and size $34.27 \times 40.29$. For both approximants, we find the order parameter $\phi \simeq 0.9998$ close to the ideal value $\phi=1$. We have no indication that the type of approximant is decisive for the results obtained below.

To speed up the computer simulations, we store the dipolar interaction energies (and the forces) in a large matrix by discretizing the set of possible distances $\mathbf{r}_{i, j}$ and use a linear interpolation scheme between the matrix entries. As an advantage, the matrix has to be calculated only once for all the simulations, and the computer memory access is usually much faster than the repeated evaluation of the original mathematical expressions. Details of the method and how to take into account the periodic boundary conditions are outlined in the appendix.

As an example, for the two approximants described above we use a $4044 \times 4754$ matrix for the energies and a $2022 \times 2377$ matrix for the forces. We find the underlying discretization of space for the energies or forces on a length scale of order $10^{-2}-10^{-3}$ to have no noticeable influence on our results.

\section{Mechanical equilibrium}

When considering the ideal quasicrystalline structure as a potential (meta-)stable state, the first question one should ask is whether this structure can be mechanically stable, i.e., whether the net forces on all the dipoles balance out or, in other words, whether the structure is a local or global minimum of the systems' potential energy [16]. While in our case for common periodic lattices, this is obvious from simple symmetry arguments, the situation is considerably more involved in the quasicrystalline structure, which is another instance of the peculiarities of this unusual type of ordering.

Note that in an infinite quasicrystal two different positions are never exactly equivalent. So the calculation of the long-range interaction with its surrounding dipoles can, strictly speaking, not be based on a finite piece of the ideal structure. Practically, however, as the dipolar interactions (1) or (2) decay as $r^{-3}$ with the distance $r$, the potential felt the dipoles will largely be dominated by their local surroundings (see also next section). In this context, it is interesting to note that in the infinite binary tiling any arbitrarily large piece of it repeats (up to rotations) within a distance of the order of its size (see e.g. [17]).

In the ideal structure, the net forces acting on a dipole converge to a relative precision of $10^{-6}$ when taking into account neighboring particles up to a distance of $r_{\max } \simeq 25$. The resulting force components clearly show that there is no dipole strength ratio $D$ which would make the ideal structure a minimum of the potential energy surface. On the other hand, when we let the ideal quasicrystalline structure relax via the method of steepest descent into a local potential minimum, we find that in the range $4 \lesssim D \lesssim 6.5$ the positions of the dipoles are only very slightly shifted. Based on the steepest descent algorithm, however, we cannot state simple systematic rules for the decoration of the binary tiling such that the dipoles assume an exact mechanical equilibrium. Due to this fact and since the energy per dipole of the relaxed configurations and the ideal quasicrystal are almost the same, we retain the decoration of the binary tiling as model reference structure.

\section{GROUND STATE CALCULATIONS}

Considering the quasicrystalline structure as a potential equilibrium state, we next investigate how this structure compares energetically to plausible alternative ordered states. To this end, we examine the energy per dipole in a binary dipolar system with the mixing ratio $x=\tau /(2+\tau)$ and the total number density of dipoles $\rho \simeq 1.231$, which are the values of the ideal quasicrystalline structure. The dipole strength ratio $D$ then remains as the only free parameter.

To determine the energy per dipole $E_{\star}$ in the ideal quasicrystalline structure, we calculate the average energy of about $10^{4}$ dipoles within a maximum distance $r_{\max }=300$ and extrapolate for $r_{\max } \rightarrow \infty$ [18]. In view of the discussion in the previous section, we should mention that the value of $E_{\star}$ may be up to a few per mille higher than the energy per dipole in the slightly 'relaxed' quasicrystal. Note that the conclusion from this section will not alter due to such differences.

The irrational mixing ratio $x$ requires alternative ordered structures based on regular lattices to be phaseseparated, i.e., to be a combination of two distinct lattices, one with a higher mixing ratio than in the quasicrystal and another one with a lower mixing ratio. In the thermodynamic limit, we can neglect energy contributions from the interfacial boundaries between the two phases and optimize their respective lattice constants (or their 'volume fractions') to minimize the total energy per particle $E_{i}$ of the two-phase state.

Some plausible alternative structures are depicted in fig. 2 ( $i$ ) two hexagonal lattices (phase separation of A and $\mathrm{B}$ dipoles), (ii) a hexagonal A lattice with $\mathrm{B}$ dipoles in the triangle centers and a hexagonal A lattice, (iii) a 


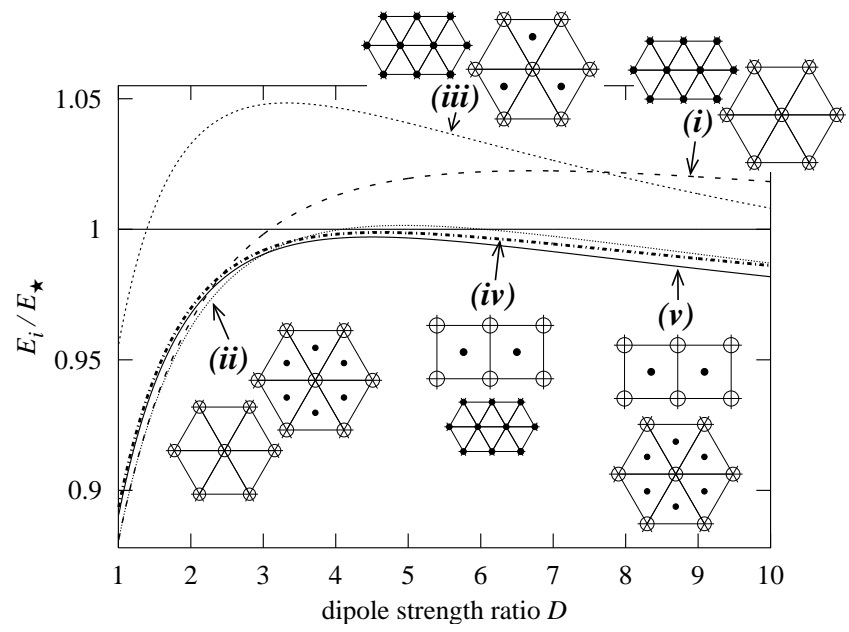

FIG. 2: Alternative ordered structures and their energies per particle $\left(E_{i}\right)$ compared to that of the quasicrystalline structure $\left(E_{\star}\right)$. Plotted is the fraction $E_{i} / E_{\star}$ as a function of the ratio of dipole moments $D$.

hexagonal A lattice with $\mathrm{B}$ dipoles in every second triangle center and a hexagonal B lattice, (iv) a centered square lattice of $\mathrm{A}$ and $\mathrm{B}$ dipoles and a hexagonal lattice of $\mathrm{B}$ dipoles, and $(v)$ a centered square lattice of $\mathrm{A}$ and $\mathrm{B}$ dipoles together with the centered $\mathrm{A}-\mathrm{B}$ hexagonal lattice of (ii). The corresponding values $E_{i}$ for dipole strength ratios $1 \leq D \leq 10$ are shown in the graph in fig. 20 relative to the value $E_{\star}$ of the quasicrystalline structure.

The figure shows the preference of hexagonal order and the degeneracy of structures (i) and (ii) for $D=1$, i.e., identical A and B dipoles. Furthermore, hexagonal ordering is preferred for $D \lesssim 3$, whereas for larger $D$, partial tetragonal ordering seems energetically favorable. The quasicrystalline structure is closest to the optimum structure in the range $4 \lesssim D \lesssim 6$, with an optimum around $D \simeq 4.5$. However, the phase-separated structure (v) clearly has lower energy. Accordingly, the quasicrystalline structure cannot be the ground state of the dipolar mixture.

Still, we find that from a purely energetic point of view the quasicrystalline structure is a surprisingly competitive type of ordering for a binary mixture of dipoles within the appropriate mixing ratio and dipole strength ratio. In view of the fact that the alternative ordered structures (i)-(v) require a phase boundary, which possesses a surface energy, the quasicrystal may become the preferred structure in finite systems. Interestingly, the quasicrystal becomes more favorable when the interaction potential $E_{\text {int }} \sim r^{-3}$ is modified to a fictitious $\tilde{E}_{\text {int }} \sim r^{-\alpha}$ with $\alpha$ close to $2(\alpha>2$ for reasons of convergence). Albeit we are not aware of any realization of such a potential in nature, this indicates that other types of scale-free interactions more strongly support the quasicrystalline ordering.

\section{STABILITY ANALYSIS OF REFERENCE STRUCTURES}

\section{A. Steepest descent calculations}

In this section, we test the dynamical stability of the quasicrystalline structure using the method of steepest descent. We displace the dipoles in small steps along their potential gradient with the step length being proportional to the modulus of the gradient vector. In numerics, this algorithm is used to find minima of multiparametric functions 19]. In physical terms, it describes the overdamped motion of the particles at zero temperature, which may be considered the simplest type of dynamics to be implemented in a system. The method has been applied before to Lennard-Jones quasicrystals [20].

In our simulations, the maximum step length (for the dipole experiencing the largest force) is limited to a fixed value of order $0.01-0.001$. Typically, after $10^{3}-10^{4}$ steps, the relaxation is finished when the dipoles start to oscillate about fixed positions, where the amplitudes are of the order of the maximum step length. We perform two types of steepest descent calculations for varying dipole strength ratio $D$. In the first one, we start from the ideal quasicrystalline positions of the dipoles, and in the second one, we perturb the ideal positions by Gaussian random noise of different strength.

The results of these simulations are somewhat hard to quantify since their concrete numerical outcome depends on the details of the algorithmic implementation, e.g., the value of the step length. The long-range dipolar potential and the lack of simple translational symmetry (which would let forces balance out trivially) give rise to a large number of dipole configurations being - mostly shallow - local energetic minima. Which of the different minima will be reached in a steepest descent calculation depends on computational details and initial conditions. Nonetheless, robust trends in the relaxation behavior are revealed and we obtain a reliable picture of the systems' dynamical stability.

\section{B. Stability as a function of dipole strength ratio $D$}

Figure 3 shows the order parameter $\phi$ as a function of the dipole strength ratio $D$ as obtained after steepest descent calculations starting from the ideal quasicrystalline structure. The data indicate that the 'relaxed' configurations stay closest to the ideal structure in a range $4.5 \leq D \leq 5.5$ with an optimum around $D \simeq 4.8$. The optimum range of $D$ essentially coincides with the range deduced from the comparison with alternative ordered structures in sec. IIII

This result is reproducible for different values of the step length and for different approximants (see fig. 3), though the values of the relaxed order parameters $\phi$ may differ. This difference is not particularly significant, since the magnitude of $\phi$ is very sensitive even to small dis- 


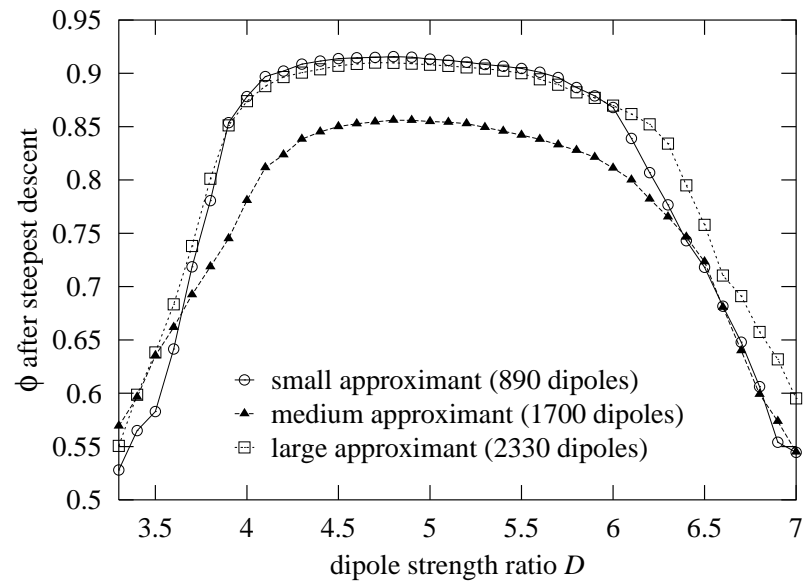

FIG. 3: Order parameter $\phi$ after steepest descent calculations starting from the ideal quasicrystalline structure as a function of the dipole strength ratio $D$ for three different approximants.

placements of the dipoles. For example, we find $\phi \simeq 0.8$ if the dipole coordinates are randomly altered with respect to their values in the ideal quasicrystalline structure by a Gaussian noise with standard deviation $\sigma=0.02$ (cf. fig. (4). Therefore, it is reasonable to consider the 'relaxed' structures to represent the ideal quasicrystalline configuration.

\section{Recovery from perturbations}

Next, we test for metastability of the structure and ask if the quasicrystalline order recovers from small perturbations. Therefore, we apply Gaussian noise with zero mean and standard deviation $\sigma$ to each particle coordinate before letting them relax via steepest descent. The average order parameter $\phi$ of the final configurations is plotted in fig. 4 as a function of $\sigma$ for a few values of $D$ from the optimum range.

Up to an average initial displacement $\sigma \lesssim 0.15$, the system relaxes back to the quasicrystalline structure, whereas for larger $\sigma$, numerous defects remain as reflected by the decreasing average order parameter $\phi$. This behavior is reminiscent of the empirical Lindemann criterion, which predicts the melting of a crystal for an average displacement of 0.14 in units of the lattice constant. We note also that due to the initial Gaussian noise the order parameter decreases below 0.1 at $\sigma=0.15$ from which it recovers its optimum value around 0.9. This means that the quasicrystalline state is relatively robust against random perturbations.

Figures 3 and 4 also include results for larger approximants. Clearly, the increasing system size does not lead to a significantly enhanced or reduced stability of the quasicrystalline order. For example, in Fig. B the order parameter at $D=4.8$ is reduced to 0.85 in the medium approximant but recovers a value above 0.9 in the large

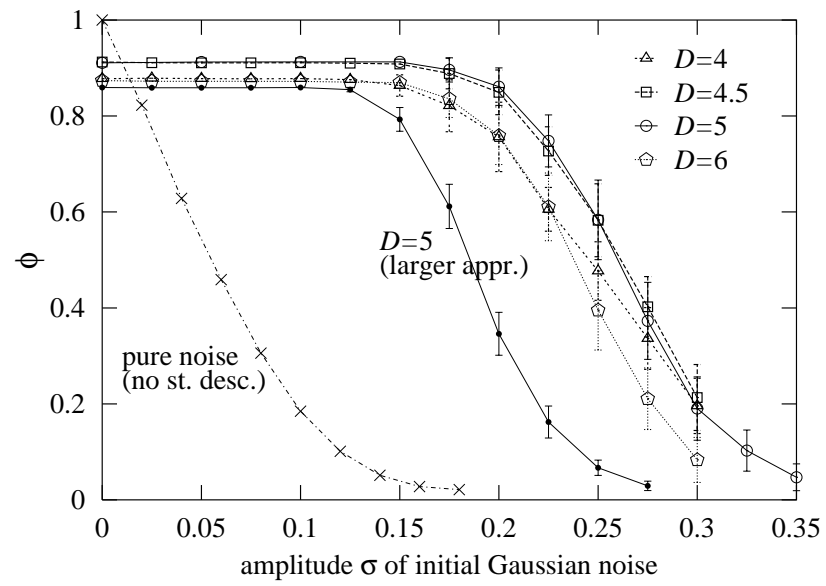

FIG. 4: Average order parameter $\phi$ after steepest descent calculations starting from a perturbed structure for different values of $D . \phi$ is plotted as a function of the standard deviation $\sigma$ of the initial Gaussian displacements. As indicated, one curve corresponds to a larger approximant (1700 instead of 890 dipoles). For comparison, the order parameter $\phi$ due to pure Gaussian noise is shown, i.e., before the steepest descent relaxation starts.

approximant. Hence we conclude that the stability of the quasicrystalline binary tiling has to be an intrinsic property of the ordered structure rather than a finite size effect.

\section{BEHAVIOR AT FINITE TEMPERATURES}

\section{A. Monte Carlo simulations}

To assess the behavior of the binary system at finite temperatures we choose Monte Carlo (MC) simulations. While computationally less costly than e.g. Langevin dynamics or even more detailed schemes, the dynamical MC simulations allow us to explore thermodynamical equilibrium states and, with a reasonable choice of jump trials, should also yield a realistic scenario of the systems' evolution.

As a jump trial in our simulations, a particle is chosen at random and imposed a Gaussian distributed displacement. The trial is accepted according to the Metropolis rule. The standard deviation of the Gaussian in the range $0.001-0.1$ is adjusted dynamically to ensure an efficient acceptance rate of trials in the range 10-60\%.

For sufficiently low temperatures, the outcome of these standard MC simulations goes well together with the results from the steepest descent simulations. For example, starting from the ideal or slightly perturbed quasicrystalline structure at $T=0.005$, we find the order parameter first to decay and then to fluctuate around $\phi \simeq 0.75$, which confirms that (nearly) quasicrystalline order represents a local energetic minimum in phase space.

However, especially at slightly higher $T$, the degree 


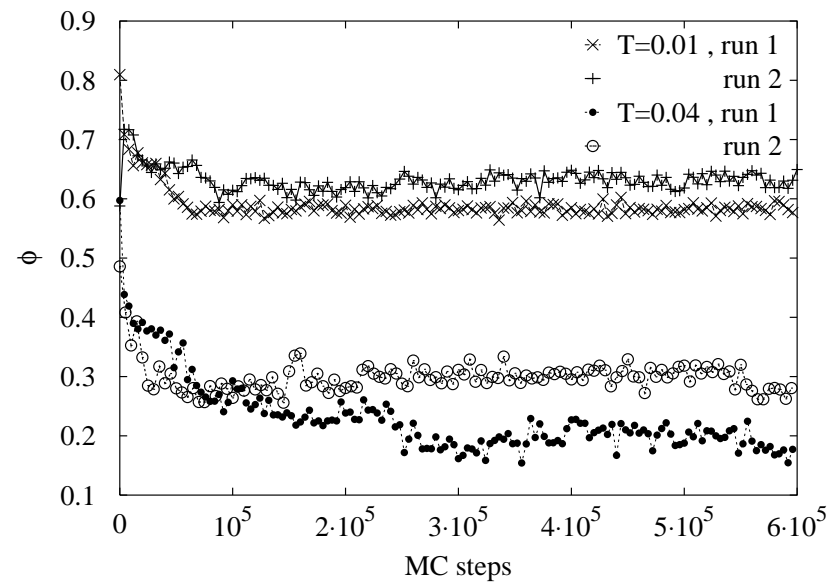

FIG. 5: Example trajectories of the order parameter $\phi$ as a function time (MC steps) from standard MC simulations at two different (fixed) temperatures $T$. The simulations are for $D=5$ in an approximant of 760 dipoles. For each $T$, 'run 1' started from the ideal quasicrystalline structure, while the initial configuration of 'run 2' was perturbed by Gaussian noise with $\sigma=0.1$ (cf. sec. IV).

and speed of the order parameter relaxation vary strongly from run to run in the MC simulations. In fig. trajectories of $\phi$ are shown for $T=0.01$ and 0.04 . The quasicrystalline structure is relatively stable at $T=0.01$, but the attained value of $\phi$ depends erratically on the initial conditions of the simulation. For example, in fig. 5 the order parameter emerging from an initially perturbed structure (run 2) unexpectedly exceeds the order parameter emerging from the ideal quasicrystalline structure (run 1). Moreover, at $T=0.04$ a slow and unpredictable decay of $\phi$ becomes observable on the time scale reached in the simulation. Even after excessively long runs of more than $10^{6} \mathrm{MC}$ steps, it remains unclear whether the system has reached an equilibrated state.

\section{B. Parallel tempering}

To accelerate thermalization in the simulations, there are different possibilities. One of them is to introduce 'artificial' multi particle flips as additional MC moves, as was previously done for the Lennard-Jones system in [6]. In an extra series of our simulations, we tested an elementary version of such flips, whereby every $100 \mathrm{MC}$ steps the positions of an $\mathrm{A}$ and a $\mathrm{B}$ dipole are interchanged and the new configuration is evolved for several MC steps before the whole move is either accepted or rejected. In general, we find the relaxation of $\phi$ to be faster, but the overall behavior remains unaltered.

For the main part of our simulations, we use the standard local moves of single particles, which might be closer to the dynamics of the experimental systems, but we apply a more sophisticated thermalization scheme known as parallel tempering [21, 22]. Basically, the idea of the method is to circumvent trapping of a systems' dynamics in local energetic minima at low temperatures by occasionally interchanging the configuration with the one of the same system simulated in parallel at higher temperatures. Here, we consider 26 copies of our system at temperatures $2.5 \times 10^{-4}=T_{1}>T_{2}>\ldots>T_{26}=0.071$. Every $2000 \mathrm{MC}$ steps, the particle configurations at adjacent temperatures $T_{i}, T_{i+1}$ are interchanged with a Metropolis type rate,

$$
w_{i, i+1}=\left\{\begin{array}{cl}
\exp \left[\left(\frac{1}{T_{i}}-\frac{1}{T_{i+1}}\right)\left(E_{i+1}-E_{i}\right)\right] & \text { if } E_{i+1}>E_{i} \\
1 & \text { else }
\end{array}\right.
$$

where $E_{i}$ and $E_{i+1}$ are the energies of the configurations. It can be shown that this scheme allows different configurations to occur with their correct Boltzmann weight at any of the temperatures $T_{i}$.

In our implementation of the algorithm, a control program on a single PC keeps track of the configurations simulated at the various temperatures $T_{i}$. Once the assignment of a certain configuration to a $T_{i}$ has been made for the next $2000 \mathrm{MC}$ steps, it is passed as independent computing job to our queuing system. So the simulations can be carried out on a variable number of available CPUs which may also differ in speed. One MC step for a single configuration takes about $0.5 \mathrm{~s}$ on a contemporary Intel Pentium IV $2.8 \mathrm{GHz}$ CPU.

We find the parallel tempering algorithm to be highly effective in thermalizing our ensemble of 26 systems. This relatively large number allows us to keep the spacing between the $T_{i}$ small and thus to change configurations frequently while still covering a large range of temperatures from possible ordering to apparently fluid-like behavior. After typically 300 rounds (i.e. $300 \times 2000 \mathrm{MC}$ steps), we see no qualitative differences any more between the extremes of an ensemble started from the ideal quasicrystalline structure and one started from random initial positions.

\section{Results: local ordering}

Figure 6 shows the mean order parameters $\phi$ and $\tilde{\phi}_{n}$ for several $n$ as a function of temperature $T$. The values are obtained from particle configurations at the respective $T_{i}$, each taken at the end of the $2000 \mathrm{MC}$ step cycles of the parallel tempering scheme. The simulations are carried out at a dipole strength ratio $D=4.75$ and started from random initial positions. The behavior is very similar for $D=4.5$ and 5. Apart from larger initial fluctuations of the order parameters $\phi$ and $\tilde{\phi}_{10}$, the same holds for simulations started from ordered initial positions.

In fig. 7 part of an example configuration from the lower temperature range in the parallel-tempering ensemble is shown. In this representative example, no longrange quasicrystalline ordering is discernable. This finding is further supported by fig. [6] The order parameter $\phi$ 


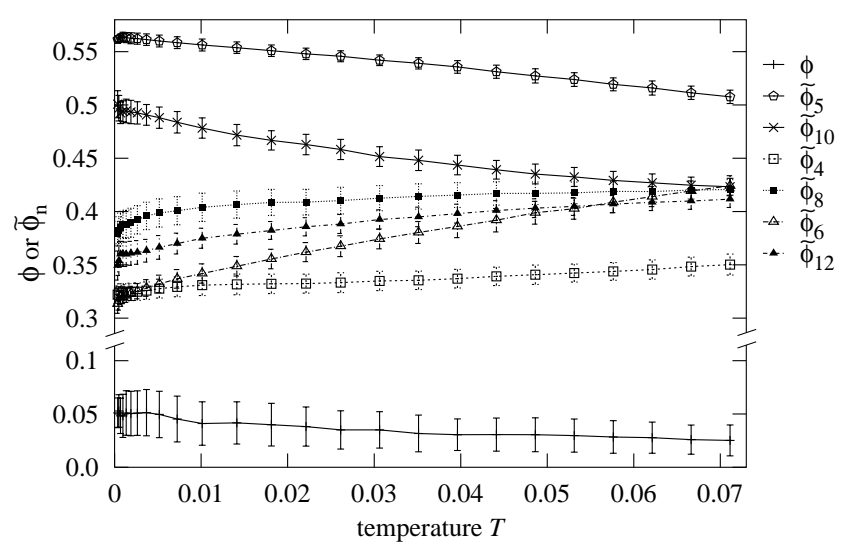

FIG. 6: Order parameters $\phi$ and $\tilde{\phi}_{n}$ for several $n$ as a function of temperature $T$ as obtained from the parallel tempering MC simulations. The dipole strength ratio is $D=4.75$. Note the jump in the ordinate.

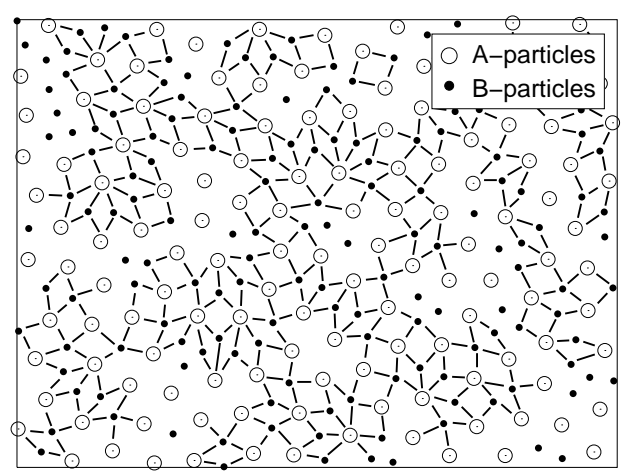

FIG. 7: Configuration from the parallel tempering MC simulations for $D=4.75$. The temperature is $T=0.00134$, the order parameter $\phi \simeq 0.07$ and energy per particle $E \simeq 8.087$. The part of the system shown is at the same scale as in fig. 11 The lines highlight local quasicrystalline ordering and are drawn whenever the bond angles between nearest neighbors of A (B) particles are even (odd) multiples of $2 \pi / 10$ (within an error of $10 \%$ ).

stays very small throughout the whole temperature range and there is no signature of a phase transition.

We find that the mean potential energy per particle in the final configurations approximately fulfills $E(T) \simeq$ $8.083+T$ at low $T$, where the simple dependence on $T$ can be understood from a harmonic approximation around the configurations with lowest energy. Thus, at low temperatures, typical configurational energies are below both the energy of the ideal quasicrystalline structure $\left(E_{\star} \simeq 8.103\right.$ for $\left.D=4.75\right)$ and the one reached in the steepest descent calculations, cf. sec. IV] On the other hand, $E$ is still above the energy of the optimum phase separated structure $\left(E_{(v)} \simeq 8.078\right)$ found in the ground state energy calculations in sec. III] This is not surprising, since the necessity of a phase boundary might prevent the dipolar mixture from reaching its possible ground state as deduced for infinite system size.

Based on these results, we can exclude the spontaneous occurrence of long-range quasicrystalline order in a thermodynamically stable phase. Moreover, irrespective of the (nearly) quasicrystalline structure being stable against small mechanical perturbations, we think that it may not represent a thermodynamically metastable state. Such a state would become separated from other states by an infinite free energy barrier in the limit of infinite system size.

In accordance with experimental observations [9, 10], we find, however, that, while the overall structure is amorphous, there occur small domains with particles arranged as in the quasicrystal, see fig. (7. As can be seen from the value of $\tilde{\phi}_{5}$ and $\phi_{10}$ compared to $\tilde{\phi}_{n}$ with $n=4,8$ and 6,12 in fig. [6] there is a tendency towards a preferred local 5 - or 10 -fold symmetry at lower temperatures.

\section{CONCLUSIONS}

Our results from the parallel tempering method provide ample evidence that the quasicrystalline binary tiling with two sorts of dipoles does not correspond to a thermodynamical equilibrium state. Nevertheless, we can identify a range for the dipole strength ratio where a long-range quasicrystalline structure corresponds to a local minimum in the potential energy landscape of the system. This local minimum has an attraction basin covering Gaussian fluctuations of the particle positions up to $15 \%$ of the distance of two neighboring $\mathrm{A}$ - and $\mathrm{B}-$ dipoles in the ideal reference structure. Our simulations, however, do not indicate that the barriers separating the local minimum from other minima increase with the system size. Therefore we do not expect that such a frozen state with quasicrystalline order exists although we cannot strictly exclude this possibility [23].

In any case, at low temperatures the times for the system to escape the local minimum by surmounting free energy barriers can become rather long. This is clearly seen in the kinetics modeled by conventional Monte Carlo simulations. Hence structures with long-range quasicrystalline order can be kinetically stable over sufficiently long time to make them an interesting subject for further study and eventual applications. What "sufficiently long" in this context means could be tested in experiments. Todays optical tweezer techniques allow colloidal particles to be placed at defined positions. Accordingly, one could prepare a quasicystalline pattern and monitor its stability. Moreover, special boundary conditions and external stimuli may support the formation of quasicrystalline structures. We also found that a modified scalefree interaction potential $\propto r^{-(2+\varepsilon)}$ leads, for $\varepsilon>0$ becoming small, to ground state energies of the quasicrystal that within numerical error bars cannot be distinguished from the most favorable phase-separated lattice structures investigated in section 
In agreement with experiments we find that even in the disordered ground state of the binary dipole system, local bond orientational order with quasicrystalline 5- and 10-fold symmetry is preferred. We made quantitative predictions for the temperature behavior of several local bond-orientation order parameters, which can be tested in experiments by using, for example, two-dimensional binary mixtures of superparamagnetic colloidal particles 8, 10, 11].

\section{Acknowledgments}

We gratefully acknowledge fruitful discussions with H. König and G. Maret and thank the Sonderforschungsbereich 513 of the Deutsche Forschungsgemeinschaft for financial support.

\section{APPENDIX: ENERGY AND FORCE CALCULATIONS IN THE SIMULATIONS}

\section{General procedure}

For an efficient calculation of the energy $E(x, y)$ of (or force $\mathbf{F}(x, y)=-\boldsymbol{\nabla} E(x, y)$ acting on) a pair of dipoles $i, j$ with distance vector $\mathbf{r}_{i, j}=(x, y)$, including their images in the periodically continued systems of the simulation box, we store the corresponding values on a fine grid of pair vectors and use a linear interpolation to obtain the values for $\mathbf{r}_{i, j}$ in the continuum. According to the "minimum image convention", possible distances in $x-$ and $y$ - direction fall in the range $-L_{x} / 2 \leq x<L_{x} / 2$ and $-L_{y} / 2 \leq y<L_{y} / 2$, where $L_{x}$ and $L_{y}$ are the lengths of the system in the $x$ - and $y$-direction. We define by $\gamma \equiv L_{x} / L_{y}$ the aspect ratio. Due to symmetry, $E(x, y)$ is an even function of $x$ and $y$, while the force components $F_{x}(x, y)=-\partial_{x} E(x, y), F_{y}(x, y)=-\partial_{y} E(x, y)$ are odd functions of $x, y$, and even functions of $y, x$, respectively.
These symmetries are used to reduce the storage needs for the matrices of energy values and force components on the grid.

For short notation, we use in this appendix $L_{y}=1$ as our length unit and $\mu_{0} m_{i} m_{j} / 4 \pi L_{y}^{3}$ as our energy unit, where $m_{i}$ and $m_{j}$ are the magnetic moments of the two dipoles with pair vector $\mathbf{r}_{i, j}$ (transformation to the units used in the main text follows after elementary rescaling). Then we have

$$
E(x, y)=\sum_{\mu, \nu=-\infty}^{\infty} \frac{1}{\left[(x+\gamma \mu)^{2}+(y+\nu)^{2}\right]^{3 / 2}} .
$$

The numerical calculation of this absolutely convergent series can be done by different means, for example by employing a two-dimensional variant of the Ewald summation [24] or a simple extrapolation scheme, cf. [18]. We applied a method developed previously in our group 25], where the series in A.1 is decomposed into an inner part for distances $\sqrt{(x+\gamma \mu)^{2}+(y+\nu)^{2}}$ smaller than a cutoff radius $r_{\mathrm{m}}$, and a remaining outer part, $E=E_{\mathrm{in}}+E_{\text {out }}$. The inner part $E_{\text {in }}$ is calculated by explicitly performing the summation, while the outer part $E_{\text {out }}$ is approximated by an integral. An analogous decomposition is done for the force components. In the following we discuss the integral approximation for the outer parts and their numerical evaluation.

\section{Integral approximation for the energy}

Defining for $\alpha=0,1$

$$
\nu_{\alpha}(\mu)= \begin{cases}\operatorname{ceil} \sqrt{r_{\mathrm{m}}^{2}-\gamma^{2} \mu^{2}} & \text { if } \gamma|\mu|<r_{\mathrm{m}} \\ \alpha & \text { else }\end{cases}
$$

where $\operatorname{ceil}(x)$ is the lowest integer number larger than or equal to $x$, we obtain

$$
\begin{aligned}
E_{\text {out }}= & \sum_{\mu=-\infty}^{\infty}\left[\sum_{\nu=-\infty}^{-\nu_{0}(\mu)}[\ldots]^{-3 / 2}+\sum_{\nu=\nu_{1}(\mu)}^{\infty}[\ldots]^{-3 / 2}\right] \\
\simeq & \int_{-\infty}^{\infty} d \mu\left[\int_{-\infty}^{-\nu_{0}(\mu)} d \nu[\ldots]^{-3 / 2}+\int_{\nu_{1}(\mu)}^{\infty} d \nu[\ldots]^{-3 / 2}+\right. \\
& \left.+\frac{1}{2}\left(\left[(x+\gamma \mu)^{2}+\left(y-\nu_{0}(\mu)\right)^{2}\right]^{-3 / 2}+\left[(x+\gamma \mu)^{2}+\left(y+\nu_{1}(\mu)\right)^{2}\right]^{-3 / 2}\right)\right] \\
\equiv & \int_{-\infty}^{\infty} d \mu f(\mu ; x, y)
\end{aligned}
$$


Here $[\ldots]$ stands for $\left[(x+\gamma \mu)^{2}+(y+\nu)^{2}\right]$. The two integrals over $\nu$ together yield

$$
\int d \nu \ldots=\frac{1}{(x+\gamma \mu)^{2}}\left(2-\frac{\nu_{0}(\mu)-y}{\sqrt{(x+\gamma \mu)^{2}+\left(y-\nu_{0}(\mu)\right)^{2}}}-\frac{\nu_{1}(\mu)+y}{\sqrt{(x+\gamma \mu)^{2}+\left(y+\nu_{1}(\mu)\right)^{2}}}\right) .
$$

Due to the piecewise definition of $\nu_{\alpha}(\mu)$, it is useful to split the remaining integral over $\mu$ in A.5 according to its boundaries, with

$$
\int_{-\infty}^{\infty} d \mu f(\mu ; x, y)=\int_{|\mu| \leq r_{\mathrm{m}} / \gamma} d \mu f(\mu ; x, y)+\int_{|\mu| \geq r_{\mathrm{m}} / \gamma} d \mu f(\mu ; x, y) \equiv E_{\text {out }, 1}+E_{\text {out }, 2} .
$$

The first part $E_{\text {out }, 1}$ is calculated analytically. With the abbreviations

it reads

$$
a_{ \pm} \equiv \sqrt{1+\left(\frac{y}{r_{\mathrm{m}} \pm x}\right)^{2}}, \quad b \pm \equiv \sqrt{1+\left(\frac{y+1}{r_{\mathrm{m}} \pm x}\right)^{2}}
$$

$$
E_{\text {out }, 1}=\frac{1}{\gamma}\left[\frac{2 r_{\mathrm{m}}}{r_{\mathrm{m}}^{2}-x^{2}}-\frac{2-a_{-}-a_{+}}{y}+\frac{2-b_{-}-b_{+}}{y+1}+\frac{2-\frac{1}{a_{-}}-\frac{1}{a_{+}}}{2 y^{2}}+\frac{2-\frac{1}{b_{-}}-\frac{1}{b_{+}}}{2(y+1)^{2}}\right] .
$$

If $y=0$, the limit $y \rightarrow 0$ should be taken explicitly to avoid numerical instabilities,

$$
\begin{aligned}
\lim _{y \rightarrow 0} E_{\text {out }, 1}=\frac{1}{\gamma}[ & 2\left(\frac{1}{r_{\mathrm{m}}-x}+\frac{1}{r_{\mathrm{m}}+x}\right)+\left(2-b_{-}-b_{+}\right)+ \\
& \left.+\frac{1}{4}\left(\frac{1}{\left(r_{\mathrm{m}}-x\right)^{2}}+\frac{1}{\left(r_{\mathrm{m}}+x\right)^{2}}\right)+\left(1-\frac{1}{2 b_{-}}-\frac{1}{2 b_{+}}\right)\right] .
\end{aligned}
$$

The second part $E_{\text {out }, 2}$ is approximated by a sum,

$$
E_{\text {out }, 2} \simeq \sum_{\mu=-\mu_{1}+1}^{\mu_{1}-1} f(\mu ; x, y)+\left(\frac{1}{2}-\varepsilon\right)\left[f\left(-\mu_{1} ; x, y\right)+f\left(\mu_{1} ; x, y\right)\right],
$$

where $\mu_{1} \equiv \operatorname{ceil}\left(r_{\mathrm{m}} / \gamma\right)$ and $\varepsilon \equiv \mu_{1}-r_{\mathrm{m}} / \gamma$. For numerical stability, here the limit $x \rightarrow 0$ of the addend with $\mu=0$ should be considered explicitly,

$$
\lim _{x \rightarrow 0} f(0 ; x, y)=\frac{1}{2}\left[\left(r_{\mathrm{m}}-y\right)^{-3}+\left(r_{\mathrm{m}}+y\right)^{-3}+\left(r_{\mathrm{m}}-y\right)^{-2}+\left(r_{\mathrm{m}}+y\right)^{-2}\right] .
$$

In the numerics, the evaluation of A.11 can conveniently be combined with the summation for the inner part $E_{\text {in }}$. For the energy matrix (and for the force calculation discussed in the next section), we use $r_{\mathrm{m}}=10$. If $E$ is to be calculated repeatedly in a simulation, $r_{\mathrm{m}} \simeq 5$ might be a reasonable choice. In ample tests in the range $0.8 \leq \gamma \leq 1.2$, we find the relative error of the integral approximation of the energy not to exceed $8 \times 10^{-4}$ for

$$
r_{\mathrm{m}}=5 \text { and } 10^{-4} \text { for } r_{\mathrm{m}}=10
$$

\section{Integral approximation for the force}

The outer part $F_{x}^{\text {out }}$ of the $x$ component force is

$$
\begin{aligned}
F_{x}^{\text {out }}= & \int_{-\infty}^{\infty} d \mu\left[\frac{1}{x+\gamma \mu}\left(\frac{y-\nu_{0}(\mu)}{\left[(x+\gamma \mu)^{2}+\left(y-\nu_{0}(\mu)\right)^{2}\right]^{3 / 2}}-\frac{y+\nu_{1}(\mu)}{\left[(x+\gamma \mu)^{2}+\left(y+\nu_{1}(\mu)\right)^{2}\right]^{3 / 2}}\right)+\right. \\
& +\frac{2}{(x+\gamma \mu)^{3}}\left(\frac{y-\nu_{0}(\mu)}{\left[(x+\gamma \mu)^{2}+\left(y-\nu_{0}(\mu)\right)^{2}\right]^{1 / 2}}-\frac{y+\nu_{1}(\mu)}{\left[(x+\gamma \mu)^{2}+\left(y+\nu_{1}(\mu)\right)^{2}\right]^{1 / 2}}\right)+ \\
& \left.+\frac{3(x+\gamma \mu)}{2}\left(\frac{1}{\left[(x+\gamma \mu)^{2}+\left(y-\nu_{0}(\mu)\right)^{2}\right]^{5 / 2}}+\frac{1}{\left[(x+\gamma \mu)^{2}+\left(y+\nu_{1}(\mu)\right)^{2}\right]^{5 / 2}}\right)\right] \\
\equiv & \int_{-\infty}^{\infty} d \mu g(\mu ; x, y) .
\end{aligned}
$$


Analogous to (A.7), the integral over $\mu$ is split into two parts, of which the first one yields

$$
\begin{aligned}
F_{x}^{\text {out }, 1}= & \frac{1}{\gamma}\left\{\frac{1}{y}\left[\frac{1}{\left|r_{\mathrm{m}}-x\right|}\left(\frac{1}{a_{-}}-a_{-}\right)-\frac{1}{\left|r_{\mathrm{m}}+x\right|}\left(\frac{1}{a_{+}}-a_{+}\right)\right]\right. \\
& -\frac{1}{y+1}\left[\frac{1}{\left|r_{\mathrm{m}}-x\right|}\left(\frac{1}{b_{-}}-b_{-}\right)-\frac{1}{\left|r_{\mathrm{m}}+x\right|}\left(\frac{1}{b_{+}}-b_{+}\right)\right] \\
& -\frac{1}{2\left|r_{\mathrm{m}}-x\right|^{3}}\left(\frac{1}{a_{-}^{3}}+\frac{1}{b_{-}^{3}}\right)+\frac{1}{2\left|r_{\mathrm{m}}+x\right|^{3}}\left(\frac{1}{a_{+}^{3}}+\frac{1}{b_{+}^{3}}\right) \\
& \left.+2\left(\frac{1}{\left(r_{\mathrm{m}}+x\right)^{2}}-\frac{1}{\left(r_{\mathrm{m}}-x\right)^{2}}\right)\right\},
\end{aligned}
$$

while the second part is approximated by a sum analogous to A.11,

$$
F_{x}^{\text {out }, 2} \simeq \sum_{\mu=-\mu_{1}+1}^{\mu_{1}-1} g(\mu ; x, y)+\left(\frac{1}{2}-\varepsilon\right)\left[g\left(-\mu_{1} ; x, y\right)+g\left(\mu_{1} ; x, y\right)\right] .
$$

Since the approximation turns out to be inaccurate in the neighborhood of $x=0$ and $x=0.5 \gamma$, it is advantageous to perform the force calculation in the ranges $|x| / \gamma \lesssim 5 \times 10^{-4}$ and $0.5-|x| / \gamma \lesssim 10^{-2}$ by explicit summation.
The corresponding expressions for the force component $F_{y}^{\text {out }}$ can be obtained from the expressions for $F_{x}^{\text {out }}$ by interchanging $x$ and $y$, replacing $\gamma$ by $1 / \gamma$, and rescaling by $1 / \gamma^{4}$, i.e. $F_{y}^{\text {out }}(x, y ; \gamma)=1 / \gamma^{4} F_{x}^{\text {out }}(y / \gamma, x / \gamma ; 1 / \gamma)$.
[1] D. Shechtman, I. Blech, D. Gratias, and J. W. Cahn, Phys. Rev. Lett. 53, 1951 (1984).

[2] H.-R. Trebin (ed.), Quasicrystals - Structure and Physical Properties (Wiley-VCH, Berlin, 2003).

[3] X. Zeng, G. Ungar, Y. Liu, V. Percec, A. E. Dulcey, and J. K. Hobbs, Nature 428, 157 (2004).

[4] M. E. Zoorob, M. D. B. Charlton, G. J. Parker, J. J. Baumberg, and M. C. Netti, Nature 404, 740 (2000).

[5] M. Notomi, H. Suzuki, T. Tamamura, and K. Edagawa, Phys. Rev. Lett. 92, 123906 (2004).

[6] M. Widom, K. J. Strandburg, and R. H. Swendsen, Phys. Rev. Lett. 58, 706 (1987).

[7] F. Lançon and L. Billard, J. Phys. France 49, 249 (1988).

[8] K. Zahn, R. Lenke, and G. Maret, Phys. Rev. Lett. 82, 2721 (1999).

[9] W. Wen, L. Zhang, and P. Sheng, Phys. Rev. Lett. 85, 5464 (2000).

[10] H. König, Ph.D. thesis, Universität Konstanz, 2003.

[11] H. Knig, K. Zahn, and G. Maret, in AIP Conference Proceedings: Slow Dynamics in Complex Systems, edited by M. Tokuyama and I. Oppenheim (February 2004, in press).

[12] M. Mézard, G. Parisi, and M. A. Virasoro, Spin Glass Theory and beyond (World Scientific, Singapore, 1987).

[13] F. Gähler, M. Baake, and M. Schlottmann, Phys. Rev. B 50, 12458 (1994).

[14] F. Lançon and L. Billard, Europhys. Lett. 2, 625 (1986).

[15] O. Entin-Wohlman, M. Kleman, and A. Pavlovitch, J. Phys. France 49, 587 (1988).

[16] Strictly speaking, zero net force can also mean a saddle point in the energy landscape.

[17] M. Baake, in: Quasicrystals, edited by J. B. Suck, M. Schreiber, and P. Häussler (Springer, Berlin 2001).

[18] When one takes into account neighboring dipoles up to a maximum distance $r_{\max }$, the energy of an individual dipole in the ideal structure scales as $E \sim$ $\int^{r_{\max }} r^{-3} r d r \sim 1 / r_{\max }$ for large $r_{\max }$. In the range $50 \leq r_{\max } \leq 300$ used by us, the above scaling relation becomes practically exact and may conveniently be used to extrapolate for $r_{\max } \rightarrow \infty$.

[19] see e.g. W. H. Press, S. A. Teukolsky, W. T. Vetterling, and B. P.Flannery, Numerical Recipes in $C$ (Cambridge University Press, Cambridge 1995).

[20] J. Roth, R. Schilling, and H.-R. Trebin, Phys. Rev. B 41, 2735 (1990).

[21] K. Hukushima and K. Nemoto, J. Phys. Soc. Jpn. 65 1604 (1996)

[22] M. E. J. Newman, G. T. Barkema, Monte Carlo Methods in Statistical Physics (Clarendon Press, Oxford 1999).

[23] Our conclusion is based on the variation of the system size in steepest descent and parallel tempering MC simulations. Strictly speaking, it would require elaborate standard MC simulations (for sufficient statistics) in a large enough variety of system sizes, which is beyond the scope of the present work.

[24] see e.g. M. P.Allen and D. J. Tildesley, Computer Simulations of Liquids (Clarendon Press, Oxford 1990).

[25] B. Rinn (private communication). 